\title{
Statistical Analysis for a Three Service Point Tandem Queue with Blocking
}

\author{
Paul R. Savariappan ${ }^{1}$, P. Chandrasekhar ${ }^{2}$, V. Mani ${ }^{2}$ and S. Thobias ${ }^{2}$ \\ ${ }^{1}$ Department of Mathematics, Luther College, Iowa-52101, USA \\ ${ }^{2}$ Department of Statistics, Loyola College, Chennai-600 034, India
}

\begin{abstract}
A maximum likelihood estimator (MLE), a consistent asymptotically normal (CAN) estimator and asymptotic confidence limits for the expected number of entities in the system in a three service point tandem queue with blocking and busy service point 1 and zero queue capacity in front of service points 2 and 3 are obtained.
\end{abstract}

Keywords: CAN estimator; Expected number of entities in the system; Multivariate central limit theorem; Slutsky theorem-tandem queue.

\section{Introduction}

Most of the studies on several queueing models are confined to only obtaining expressions for transient or stationary (steady state) solutions and do not consider the associated statistical inference problems. Nowadays, statistical analysis of queueing systems is an important area of research in Queueing theory. Parametric estimation, Interval estimation and Bayesian estimation are some of the essential tools to understand any random phenomena using stochastic models. Analysis of queueing systems in this direction has not received much attention in the past. Whenever the systems are fully observable in terms of their random components such as interarrival times and service times, standard parametric techniques of statistical theory are quite appropriate. Recently, Narayan Bhat (2003) has provided an overview of methods available for estimation, when the information is restricted to the number of entities in the system at some discrete points in time. Narayan Bhat (2003) has also described how maximum likelihood estimation is applied directly to the underlying Markov chain in the queue length process in $\mathrm{M} / \mathrm{G} / 1$ and $\mathrm{GI} / \mathrm{M} / 1$ queues. Table 1 indicates the present state of work of queueing systems, wherein the asymptotic confidence limits for measures of system performance are obtained.

Generally speaking, the queueing models assume that each service channel consists of only one service point. Situations do exist, where each service channel may consist of several service points in series. In this situation, an 
entity must pass through all these service points in succession before completing its service. Such situations are known as queues in series or tandem queues. e.g., (a) in a manufacturing process, units must pass through a series of service points (work stations), where each service point performs a given task or job, (b) in a university registration process, each registrant must pass through a series of counters such as advisor, department chairman and cashier, (c) in a clinical physical examination procedure, a patient must go through a series of stages such as laboratory tests, ECG and chest X-ray. In all these models, it is not only sufficient to know how many entities are there in the system but also where they are.

Table 1: Present state of work for queueing systems

\begin{tabular}{|c|c|c|c|}
\hline $\begin{array}{l}\text { S. } \\
\text { No. }\end{array}$ & System Description & Authors & $\begin{array}{l}\text { Confidence } \\
\text { limits } \\
\text { obtained for }\end{array}$ \\
\hline 1 & $\mathrm{M} / \mathrm{M} / 1$ & Clarke (1957) & $\begin{array}{r}\text { MLEs of } \lambda \\
\text { and } \mu .\end{array}$ \\
\hline 2 & $\mathrm{M} / \mathrm{M} / 1 / \infty$ and $\mathrm{M} / \mathrm{M} / 1 / \mathrm{N}$ & Yadavalli et al (2004) & $\mathrm{W}_{\mathrm{Q}}$ \\
\hline 3 & $\mathrm{M} / \mathrm{M} / \mathrm{c} / \infty$ and $\mathrm{M} / \mathrm{M} / \mathrm{c} / \mathrm{N}$ & Yadavalli et al (2006) & $\mathrm{W}_{\mathrm{Q}}$ \\
\hline 4 & $\begin{array}{l}\text { Tandem queue with } \\
\text { blocking and dependent } \\
\text { structure for service times }\end{array}$ & $\begin{array}{l}\text { Chandrasekhar et al } \\
\text { (2006) }\end{array}$ & $\mathrm{L}_{\mathrm{s}}, \mathrm{W}_{\mathrm{s}}$ \\
\hline 5 & $\begin{array}{l}\text { Tandem queue with } \\
\text { blocking }\end{array}$ & $\begin{array}{l}\text { Chandrasekhar et al } \\
(2008)\end{array}$ & $\mathrm{W}_{\mathrm{s}}$ \\
\hline 6 & $\begin{array}{l}\text { Bulk-arrival queueing } \\
\text { system } \mathrm{M}^{[\mathrm{x}]} / \mathrm{M} / 1\end{array}$ & $\begin{array}{l}\text { Paul Savariappan and } \\
\text { Chandrasekhar (2009a) }\end{array}$ & $\mathrm{L}_{\mathrm{s}}$ \\
\hline 7 & $\begin{array}{l}\text { Tandem and bulk service } \\
\text { queueing systems }\end{array}$ & $\begin{array}{l}\text { Paul Savariappan et al } \\
(2009 \text { b) }\end{array}$ & $\mathrm{L}_{\mathrm{s}}$ \\
\hline 8 & $\begin{array}{l}\mathrm{M} / \mathrm{M} / \mathrm{R} \text { queue with } \\
\text { heterogeneous servers }\end{array}$ & $\begin{array}{l}\text { Tsung-Yin Wang et al } \\
(2006)\end{array}$ & $\mathrm{L}_{\mathrm{s}}$ \\
\hline 9 & $\begin{array}{l}\text { Two station and three } \\
\text { station tandem queues } \\
\text { with blocking }\end{array}$ & $\begin{array}{l}\text { Chandrasekhar and } \\
\text { Vaidyanathan (2009) }\end{array}$ & $\mathrm{L}_{\mathrm{s}}$ \\
\hline
\end{tabular}

In general, in all the above mentioned tandem queueing models studied so far, it is assumed that no queues are allowed in front of service points. This is not so in any real life situation. Hence, an attempt is made in this paper to study in detail a 
three service point tandem queue, where service point 1 is busy but no queue is allowed at service point 2 and service point 3. A MLE, CAN and asymptotic confidence limits for the expected number of entities in the system are also obtained.

\section{System Description and Assumptions}

Consider a simplified one channel queueing system consisting of three series service points as below :

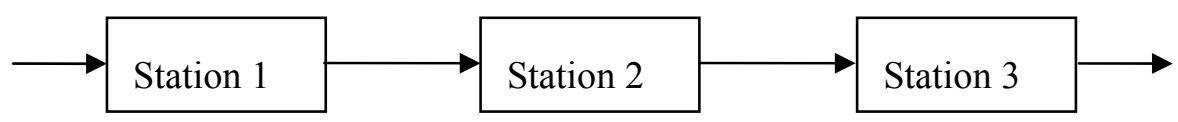

Figure 1: System configuration.

An entity arriving for service must pass through service point 1 , service point 2 and service point 3 before completing its service. The assumptions of the model are as follows:

i. Service times at service point 1 , service point 2 and service point 3 are independent and exponentially distributed with service rates $\mu_{1}, \mu_{2}$ and $\mu_{3}$ respectively.

ii. Service point 1 is busy but no queue is allowed at service point 2 and service point 3.

iii. Service point 2 and service point 3 are either free or busy.

iv. Service point $\mathrm{i}(\mathrm{i}=1,2)$ is said to be blocked, if the entity in service point $\mathrm{i}(\mathrm{i}=1,2)$ completes its service before service point $(i+1)(i=1,2)$ becomes free. In this case, the entity cannot wait between the service points $i$ and $(i+1)(i=1,2)$, since this is not allowed and the entity remains in service point $\mathrm{i}(\mathrm{i}=1,2)$ itself.

\section{Analysis of the System}

Let the symbols 0,1 , and $\mathrm{b}$ represent free, busy and blocked states of a service point. Let $X(\mathrm{t}), Y(\mathrm{t})$ and $Z(\mathrm{t})$ respectively denote the states of service point 1 , service point 2 and service point 3 and the vector process $W(\mathrm{t})=\{(X(\mathrm{t}), Y(\mathrm{t}), Z(\mathrm{t})), \mathrm{t} \geq 0\}$ with state space 


$$
\mathrm{E}=\{(1,0,0),(1,1,0),(1,0,1),(1,1,1),(\mathrm{b}, 1,0),(1, \mathrm{~b}, 1),(\mathrm{b}, 1,1),(\mathrm{b}, \mathrm{b}, 1)\}
$$

the state of the system at time t. Since the interarrival and service times are all exponential, it clearly follows that the process $\mathrm{W}(\mathrm{t})$ is a Markov process with the infinitesimal generator given by

$$
\begin{aligned}
& (1,0,0) \quad(1,1,0) \quad(1,0,1) \quad(1,1,1) \quad(\mathrm{b}, 1,0) \quad(1, \mathrm{~b}, 1) \quad(\mathrm{b}, 1,1) \quad(\mathrm{b}, \mathrm{b}, 1) \\
& \begin{array}{c}
(1,0,0) \\
(1,1,0)
\end{array}\left[\begin{array}{cccccccc}
-\mu_{1} & \mu_{1} & 0 & 0 & 0 & 0 & 0 & 0 \\
0 & -\left(\mu_{1}+\mu_{2}\right) & \mu_{2} & 0 & \mu_{1} & 0 & 0 & 0
\end{array}\right]
\end{aligned}
$$

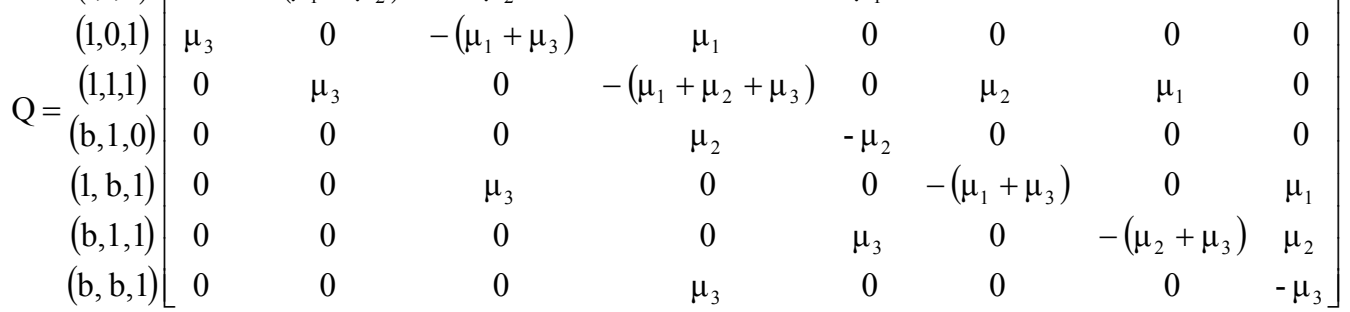

Let

$\mathrm{a}=(1,0,0), \mathrm{b}^{\prime}=(1,1,0), \mathrm{c}=(1,0,1), \mathrm{d}=(1,1,1), \mathrm{e}=(\mathrm{b}, 1,0), \mathrm{f}=(1, \mathrm{~b}, 1), \mathrm{g}=(\mathrm{b}, 1,1)$ and $\mathrm{h}=(\mathrm{b}, \mathrm{b}, 1)$. Also, let $\mathrm{p}_{\mathrm{ijk}}(\mathrm{t})=\operatorname{Pr}[\mathrm{W}(\mathrm{t})=(\mathrm{i}, \mathrm{j}, \mathrm{k})] \forall(\mathrm{i}, \mathrm{j}, \mathrm{k}) \in \mathrm{E}$ represent the probability that the system is in state $(\mathrm{i}, \mathrm{j}, \mathrm{k})$ at time $\mathrm{t}$ with the initial condition $\mathrm{p}_{100}(0)=1$. From the infinitesimal generator given in (3.2), we have the following system of differential - difference equations:

$$
\begin{gathered}
\frac{d p_{a}(t)}{d t}=-\mu_{1} p_{a}(t)+\mu_{3} p_{c}(t) \\
\frac{d p_{b^{\prime}}(t)}{d t}=\mu_{1} p_{a}(t)-\left(\mu_{1}+\mu_{2}\right) p_{b}(t)+\mu_{3} p_{d}(t) \\
\frac{d p_{c}(t)}{d t}=\mu_{2} p_{b}(t)-\left(\mu_{1}+\mu_{3}\right) p_{c}(t)+\mu_{3} p_{f}(t) \\
\frac{d p_{d}(t)}{d t}=\mu_{1} p_{c}(t)-\left(\mu_{1}+\mu_{2}+\mu_{3}\right) p_{d}(t)+\mu_{2} p_{e}(t)+\mu_{3} p_{h}(t) \\
\frac{d p_{e}(t)}{d t}=\mu_{1} p_{b}(t)-\mu_{2} p_{e}(t)+\mu_{3} p_{g}(t) \\
\frac{d p_{f}(t)}{d t}=\mu_{2} p_{d}(t)-\left(\mu_{1}+\mu_{3}\right) p_{f}(t)
\end{gathered}
$$




$$
\begin{gathered}
\frac{d p_{g}(t)}{d t}=\mu_{1} p_{d}(t)-\left(\mu_{2}+\mu_{3}\right) p_{g}(t) \\
\frac{d p_{h}(t)}{d t}=\mu_{1} p_{f}(t)+\mu_{2} p_{g}(t)-\mu_{3} p_{h}(t)
\end{gathered}
$$

\subsection{Steady state solution}

The equations (3.3) - (3.10) can be solved using the fact that $\sum_{(\mathrm{i}, \mathrm{j}, \mathrm{k}) \in \mathrm{E}} \mathrm{p}_{\mathrm{ijk}}(\mathrm{t})=1$. Since we wish to study the stationary behaviour of the system, let $\lim _{t \rightarrow \infty} p_{i j k}(t)=p_{i j k}$.

Let $\underline{p}=\left(p_{a}, p_{b^{\prime}}, p_{c}, p_{d}, p_{e}, p_{f}, p_{g}, p_{h}\right)$ be the stationary distribution corresponding to the Markov process $\{\mathrm{W}(\mathrm{t}), \mathrm{t} \geq 0\}$. The steady state probabilities are given by

$$
\begin{aligned}
& \mathrm{p}_{\mathrm{a}}=\frac{\mu_{2}^{2} \mu_{3}^{3}\left(\mu_{1}+\mu_{2}\right)\left(\mu_{2}+\mu_{3}\right)\left(2 \mu_{1}+\mu_{2}+\mu_{3}\right)}{\left\{\left(\mu_{1}+\mu_{2}+\mu_{3}\right) f\left(\mu_{1}, \mu_{2}, \mu_{3}\right)+g\left(\mu_{1}, \mu_{2}, \mu_{3}\right)\right\}} \\
& \mathrm{p}_{\mathrm{b}^{\prime}}=\frac{\left.\mu_{1} \mu_{2} \mu_{3}^{2}\left(\mu_{2}+\mu_{3}\right)\left(\mu_{1}+\mu_{2}+\mu_{3}\right)\left(\mu_{1}^{2}+\mu_{2} \mu_{3}+\mu_{3} \mu_{1}\right)+\mu_{1} \mu_{2} \mu_{3}\right]}{\left\{\left(\mu_{1}+\mu_{2}+\mu_{3}\right) \mathrm{f}\left(\mu_{1}, \mu_{2}, \mu_{3}\right)+\mathrm{g}\left(\mu_{1}, \mu_{2}, \mu_{3}\right)\right\}} \\
& p_{c}=\frac{\mu_{1} \mu_{2}^{2} \mu_{3}^{2}\left(2 \mu_{1}+\mu_{2}+\mu_{3}\right)\left(\mu_{1}+\mu_{2}\right)\left(\mu_{2}+\mu_{3}\right)}{\left\{\left(\mu_{1}+\mu_{2}+\mu_{3}\right) f\left(\mu_{1}, \mu_{2}, \mu_{3}\right)+g\left(\mu_{1}, \mu_{2}, \mu_{3}\right)\right\}} \\
& \mathrm{p}_{\mathrm{d}}=\frac{\mu_{1}^{2} \mu_{2} \mu_{3}\left(\mu_{1}+\mu_{2}\right)\left(\mu_{2}+\mu_{3}\right)\left(\mu_{3}+\mu_{1}\right)\left(\mu_{1}+\mu_{2}+\mu_{3}\right)}{\left\{\left(\mu_{1}+\mu_{2}+\mu_{3}\right) \mathrm{f}\left(\mu_{1}, \mu_{2}, \mu_{3}\right)+\mathrm{g}\left(\mu_{1}, \mu_{2}, \mu_{3}\right)\right\}} \\
& \mathrm{p}_{\mathrm{e}}=\frac{\mu_{1}^{2} \mu_{3}^{2}\left[\mu_{2} \mu_{3}\left(\mu_{2}+\mu_{3}\right)\left(2 \mu_{1}+\mu_{2}+\mu_{3}\right)+\mu_{1}\left(\mu_{1}+\mu_{3}\right)\left(\mu_{1}+\mu_{2}+\mu_{3}\right)\left(\mu_{1}+2 \mu_{2}+\mu_{3}\right)\right]}{\left\{\left(\mu_{1}+\mu_{2}+\mu_{3}\right) \mathrm{f}\left(\mu_{1}, \mu_{2}, \mu_{3}\right)+\mathrm{g}\left(\mu_{1}, \mu_{2}, \mu_{3}\right)\right\}} \\
& \mathrm{p}_{\mathrm{f}}=\frac{\mu_{1}^{2} \mu_{2}^{2} \mu_{3}\left(\mu_{1}+\mu_{2}\right)\left(\mu_{2}+\mu_{3}\right)\left(\mu_{1}+\mu_{2}+\mu_{3}\right)}{\left\{\left(\mu_{1}+\mu_{2}+\mu_{3}\right) \mathrm{f}\left(\mu_{1}, \mu_{2}, \mu_{3}\right)+\mathrm{g}\left(\mu_{1}, \mu_{2}, \mu_{3}\right)\right\}}
\end{aligned}
$$




$$
\begin{aligned}
& p_{g}=\frac{\mu_{1}^{3} \mu_{2} \mu_{3}\left(\mu_{1}+\mu_{2}\right)\left(\mu_{3}+\mu_{1}\right)\left(\mu_{1}+\mu_{2}+\mu_{3}\right)}{\left\{\left(\mu_{1}+\mu_{2}+\mu_{3}\right) f\left(\mu_{1}, \mu_{2}, \mu_{3}\right)+g\left(\mu_{1}, \mu_{2}, \mu_{3}\right)\right\}} \\
& p_{h}=\frac{\mu_{1}^{3} \mu_{2}^{2}\left(\mu_{1}+\mu_{2}\right)\left(\mu_{1}+\mu_{2}+\mu_{3}\right)\left(\mu_{1}+\mu_{2}+2 \mu_{3}\right)}{\left\{\left(\mu_{1}+\mu_{2}+\mu_{3}\right) f\left(\mu_{1}, \mu_{2}, \mu_{3}\right)+g\left(\mu_{1}, \mu_{2}, \mu_{3}\right)\right\}}
\end{aligned}
$$

where

$$
\begin{aligned}
& \mathrm{f}\left(\mu_{1}, \mu_{2}, \mu_{3}\right)=\left(\mu_{1}+\mu_{2}\right)\left\{\mu_{2}^{2} \mu_{3}\left(\mu_{2}+\mu_{3}\right)\left(\mu_{1}^{2}+\mu_{1} \mu_{3}+\mu_{3}^{2}\right)\right. \\
& \left.+\mu_{1}^{2} \mu_{2} \mu_{3}\left(\mu_{3}+\mu_{1}\right)\left(\mu_{1}+\mu_{2}+\mu_{3}\right)+\mu_{1}^{3} \mu_{2}^{2}\left(\mu_{1}+\mu_{2}+2 \mu_{3}\right)\right\}+ \\
& \mu_{1} \mu_{2} \mu_{3}^{2}\left(\mu_{2}+\mu_{3}\right)\left(\mu_{1}^{2}+2 \mu_{1} \mu_{3}+\mu_{2} \mu_{3}\right)+\mu_{1}^{3} \mu_{3}^{2}\left(\mu_{3}+\mu_{1}\right)\left(\mu_{1}+2 \mu_{2}+\mu_{3}\right)
\end{aligned}
$$

and

$$
\mathrm{g}\left(\mu_{1}, \mu_{2}, \mu_{3}\right)=\mu_{1} \mu_{2} \mu_{3}^{2}\left(\mu_{1}+\mu_{2}\right)\left(\mu_{2}+\mu_{3}\right)\left[\mu_{1} \mu_{2}+\mu_{2} \mu_{3}+\mu_{3} \mu_{1}\right]
$$

\subsection{Expected number of entities in the system}

The expected number of entities in the system is given by

$$
\mathrm{L}_{\mathrm{s}}=\sum_{\mathrm{n}=\mathrm{o}}^{\infty} \mathrm{n} \cdot \mathrm{p}_{\mathrm{n}}=1\left(\mathrm{p}_{\mathrm{a}}\right)+2\left(\mathrm{p}_{\mathrm{b}^{\prime}}+\mathrm{p}_{\mathrm{c}}+\mathrm{p}_{\mathrm{e}}\right)+3\left(\mathrm{p}_{\mathrm{d}}+\mathrm{p}_{\mathrm{f}}+\mathrm{p}_{\mathrm{g}}+\mathrm{p}_{\mathrm{h}}\right)
$$

Substituting for $\mathrm{p}_{\mathrm{a}}, \mathrm{p}_{\mathrm{b}^{\prime}}, \mathrm{p}_{\mathrm{c}}, \mathrm{p}_{\mathrm{d}}, \mathrm{p}_{\mathrm{e}}, \mathrm{p}_{\mathrm{f}}, \mathrm{p}_{\mathrm{g}}, \mathrm{p}_{\mathrm{h}}$, it can be shown that

$$
\mathrm{L}_{\mathrm{s}}=\frac{\left\{\left(\mu_{1}+\mu_{2}+\mu_{3}\right) \mathrm{f}_{1}\left(\mu_{1}, \mu_{2}, \mu_{3}\right)+\mathrm{g}_{1}\left(\mu_{1}, \mu_{2}, \mu_{3}\right)\right\}}{\left\{\left(\mu_{1}+\mu_{2}+\mu_{3}\right) \mathrm{f}\left(\mu_{1}, \mu_{2}, \mu_{3}\right)+\mathrm{g}\left(\mu_{1}, \mu_{2}, \mu_{3}\right)\right\}}
$$

where

$$
\begin{gathered}
\mathrm{f}_{1}\left(\mu_{1}, \mu_{2}, \mu_{3}\right)=\left\{\left(\mu_{2}+\mu_{3}\right)\left[\mu_{2}^{2} \mu_{3}^{3}\left(\mu_{1}+\mu_{2}\right)+2 \mu_{1}^{2} \mu_{2} \mu_{3}^{2}\left(\mu_{3}+\mu_{1}\right)+2 \mu_{1}^{2} \mu_{2} \mu_{3}^{3}\right]+2 \mu_{1}^{3} \mu_{3}^{2} \mu_{2}\left(\mu_{3}+\mu_{1}\right)\right\} \\
+\left(\mu_{1}+\mu_{2}+\mu_{3}\right)\left[3 \mu_{1}^{3} \mu_{2}^{2}\left(\mu_{1}+\mu_{2}\right)+2 \mu_{1} \mu_{2}^{2} \mu_{3}^{2}\left(\mu_{2}+\mu_{3}\right)+2 \mu_{3}^{2} \mu_{1}^{3}\left(\mu_{3}+\mu_{1}\right)\right] \\
+3 \mu_{1}^{2} \mu_{2} \mu_{3}\left(\mu_{1}+\mu_{2}\right)\left(\mu_{1}+\mu_{2}+\mu_{3}\right)^{2}
\end{gathered}
$$

and

$$
\mathrm{g}_{1}\left(\mu_{1}, \mu_{2}, \mu_{3}\right)=\mu_{1} \mu_{2} \mu_{3}^{2}\left(\mu_{2}+\mu_{3}\right)\left\{\mu_{2}\left(\mu_{1}+\mu_{2}\right)\left(2 \mu_{1}+\mu_{3}\right)+2 \mu_{1} \mu_{3}\left(\mu_{1}+\mu_{2}\right)\right\} \text {. }
$$

Further, $\mathrm{f}\left(\mu_{1}, \mu_{2}, \mu_{3}\right)$ and $\mathrm{g}\left(\mu_{1}, \mu_{2}, \mu_{3}\right)$ are given in (3.19) and (3.20). 


\section{Special Case}

By choosing $\mu_{1}=\mu_{2}=\mu_{3}=\mu$ (say), i.e., the service times at service point 1 , service point 2 and service point 3 are independent and identically distributed (i.i.d) exponential variates with the parameter $\mu$, it is readily seen that

$$
\begin{aligned}
& \mathrm{p}_{\mathrm{a}}=\frac{4}{39}, \mathrm{p}_{\mathrm{b}^{\prime}}=\frac{5}{39}, \mathrm{p}_{\mathrm{c}}=\frac{4}{39}, \mathrm{p}_{\mathrm{d}}=\frac{6}{39}, \mathrm{p}_{\mathrm{e}}=\frac{8}{39}, \mathrm{p}_{\mathrm{f}}=\frac{3}{39}, \mathrm{p}_{\mathrm{g}}=\frac{3}{39}, \\
& \mathrm{p}_{\mathrm{h}}=\frac{6}{39} \text { and } \mathrm{L}_{\mathrm{s}}=\frac{92}{39}
\end{aligned}
$$

In the next section, we obtain the MLE and CAN for the expected number of entities in the system.

\subsection{MLE and CAN Estimator for the Expected Number of Entities in the System}

\subsubsection{Estimator}

Let $\mathrm{Y}_{11}, \mathrm{Y}_{12}, \ldots, \mathrm{Y}_{1 \mathrm{n}} ; \mathrm{Y}_{21}, \mathrm{Y}_{22}, \ldots, \mathrm{Y}_{2 \mathrm{n}} ; \mathrm{Y}_{31}, \mathrm{Y}_{32}, \ldots, \mathrm{Y}_{3 \mathrm{n}}$ be three random samples of size $n$, each drawn from three different exponential service time populations with the parameters $\mu_{1}, \mu_{2}$ and $\mu_{3}$ respectively. It is clear that $\mathrm{E}\left(\overline{\mathrm{Y}}_{\mathrm{i}}\right)=\frac{1}{\mu_{\mathrm{i}}}, \mathrm{i}=1,2,3$ where $\overline{\mathrm{Y}}_{\mathrm{i}}=\sum_{\mathrm{j}=1}^{\mathrm{n}} \mathrm{Y}_{\mathrm{ij}} / n, \mathrm{i}=1,2,3$ and $\overline{\mathrm{Y}}_{1}, \overline{\mathrm{Y}}_{2}$, and $\overline{\mathrm{Y}}_{3}$ are the sample means of service times at service point 1 , service point 2 and service point 3 respectively. It can be shown that $\bar{Y}_{i}, i=1,2,3$ is the MLE of $\frac{1}{\mu_{i}}, i=1,2,3$. Let $\theta_{1}=\frac{1}{\mu_{1}}, \theta_{2}=\frac{1}{\mu_{2}}$ and $\theta_{3}=\frac{1}{\mu_{3}}$. Clearly, the expected number of entities in the system given in (3.21) yields

$$
\mathrm{L}_{\mathrm{s}}=\ell_{1} / \ell_{2}
$$

where

$$
\begin{aligned}
& \left.\left.\ell_{1}=\theta_{1}^{3} \theta_{2} \theta_{3}\left(\theta_{1}+\theta_{2}\right)\left(\theta_{2}+\theta_{3}\right)\left(\theta_{1}+2 \theta_{2}+2 \theta_{3}\right)+\theta_{1}\left(\theta_{2}+\theta_{3}\right) \theta_{1}^{3}\left(\theta_{1}+\theta_{2}\right)+2 \theta_{1} \theta_{2}^{2}\left(\theta_{1}+\theta_{3}\right)+2 \theta_{1}^{2} \theta_{2}^{2}\right]+2 \theta_{2}^{3} \theta_{3}\left(\theta_{1}+\theta_{3}\right)\right\} \\
& \left(\theta_{1} \theta_{2}+\theta_{2} \theta_{3}+\theta_{3} \theta_{1}\right)+\left(\theta_{1} \theta_{2}+\theta_{2} \theta_{3}+\theta_{3} \theta_{1}\right)^{2}\left[2 \theta_{1}^{3}\left(\theta_{2}+\theta_{3}\right)+2 \theta_{2}^{3}\left(\theta_{3}+\theta_{1}\right)+3 \theta_{3}^{3}\left(\theta_{1}+\theta_{2}\right)\right] \\
& +3 \theta_{3}\left(\theta_{1}+\theta_{2}\right)\left(\theta_{1} \theta_{2}+\theta_{2} \theta_{3}+\theta_{3} \theta_{1}\right)^{3}
\end{aligned}
$$

and 


$$
\begin{aligned}
& \ell_{2}=\left(\theta_{1} \theta_{2}+\theta_{2} \theta_{3}+\theta_{3} \theta_{1}\right)\left\{\left(\theta_{1}+\theta_{2}\right)\left[\theta_{1}^{2}\left(\theta_{2}+\theta_{3}\right)\left(\theta_{1}^{2}+\theta_{1} \theta_{3}+\theta_{3}^{2}\right)+\theta_{2} \theta_{3}\left(\theta_{1}+\theta_{3}\right)\left(\theta_{1} \theta_{2}+\theta_{2} \theta_{3}+\theta_{3} \theta_{1}\right)+\theta_{3}^{3}\left(2 \theta_{1} \theta_{2}+\theta_{2} \theta_{3}+\theta_{3} \theta_{1}\right)\right]\right. \\
& \left.+\theta_{1}^{2} \theta_{2}\left(\theta_{2}+\theta_{3}\right)\left(2 \theta_{1} \theta_{2}+\theta_{2} \theta_{3}+\theta_{1}^{2}\right)+\theta_{2}^{3}\left(\theta_{1}+\theta_{3}\right)\left(\theta_{1} \theta_{2}+\theta_{2} \theta_{3}+2 \theta_{3} \theta_{1}\right)\right\}+\theta_{1}^{3} \theta_{2} \theta_{3}\left(\theta_{1}+\theta_{2}\right)\left(\theta_{2}+\theta_{3}\right)\left(\theta_{1}+\theta_{2}+\theta_{3}\right)
\end{aligned}
$$

and hence MLE of $\mathrm{L}_{\mathrm{s}}$ is obtained by replacing $\theta_{1}, \theta_{2}$ and $\theta_{3}$ by the corresponding estimators namely $\overline{\mathrm{Y}}_{1}, \overline{\mathrm{Y}}_{2}$ and $\overline{\mathrm{Y}}_{3}$ respectively. It may be noted that $\hat{\mathrm{L}}_{\mathrm{S}}$ is a real valued function in $\overline{\mathrm{Y}}_{\mathrm{i}},(\mathrm{i}=1,2,3)$, which is also differentiable.

\section{Special cases:}

(i) Suppose $\theta_{1}=\theta_{2}=\theta_{3}=\theta$ (say).i.e., the service times at service point 1, service point 2 and service point 3 are i.i.d exponential variates each with the parameter $\theta$, it is seen from (3.23) that $\mathrm{L}_{\mathrm{S}}=\frac{92}{39}$, which is in agreement with (3.22).

(ii) Suppose $\theta_{i}=i \theta, i=1,2,3$. From (3.23), it is further seen that

$$
\mathrm{L}_{\mathrm{S}}=\frac{79761}{29118}=2.7392 \text {. }
$$

(iii) Finally, if $\theta_{i}=(3-i) \theta, i=1,2,3$, the expected number of customers in the system reduces from $(3.23)$ to $\mathrm{L}_{\mathrm{S}}=\frac{10}{7}$.

\subsubsection{A CAN estimator using the multivariate central limit theorem}

Suppose $\mathrm{T}_{1}{ }^{\prime}, \mathrm{T}_{2}{ }^{\prime}, \mathrm{T}_{3}{ }^{\prime}, \ldots$ are independent and identically distributed $\mathrm{k}$ dimensional random variables such that

$$
T_{\mathrm{n}}{ }^{\prime}=\left(\mathrm{T}_{1 \mathrm{n}}, \mathrm{T}_{2 \mathrm{n}}, \mathrm{T}_{3 \mathrm{n}}, \ldots, \mathrm{T}_{\mathrm{kn}}\right), \mathrm{n}=1,2,3, \ldots
$$

having the first and second order moments $\mathrm{E}\left(\mathrm{T}_{\mathrm{n}}\right)=\mu$ and $\operatorname{var}\left(\mathrm{T}_{\mathrm{n}}\right)=\sum$.

Define the sequence of random variables

$$
\bar{T}_{\mathrm{n}}^{\prime}=\left(\overline{\mathrm{T}}_{1 \mathrm{n}}, \overline{\mathrm{T}}_{2 \mathrm{n}}, \overline{\mathrm{T}}_{3 \mathrm{n}}, \ldots, \overline{\mathrm{T}}_{\mathrm{kn}}\right), \mathrm{n}=1,2,3, \ldots
$$




$$
\text { where } \bar{T}_{i n}=\frac{\sum_{j=1}^{n} T_{i j}}{n}, i=1,2,3, \ldots, k \text {. }
$$

Then, $\sqrt{\mathrm{n}}\left(\overline{\mathrm{T}}_{\mathrm{n}}-\mu\right) \stackrel{\mathrm{d}}{\longrightarrow} \mathrm{N}_{\mathrm{k}}\left(0, \sum\right)$ as $\mathrm{n} \rightarrow \infty$.

By applying the multivariate central limit theorem given above, it is readily seen that

$$
\sqrt{\mathrm{n}}\left[\left(\overline{\mathrm{Y}}_{1}, \overline{\mathrm{Y}}_{2}, \overline{\mathrm{Y}}_{3}\right)-\left(\theta_{1}, \theta_{2}, \theta_{3}\right)\right] \stackrel{\mathrm{d}}{\longrightarrow} \mathrm{N}_{3}\left(0, \sum\right) \text { as } \mathrm{n} \rightarrow \infty
$$

where the dispersion matrix $\sum=\left(\left(\sigma_{\mathrm{ij}}\right)\right)$ is given by $\sum=\operatorname{diag}\left(\theta_{1}^{2}, \theta_{2}^{2}, \theta_{3}^{2}\right)$.

Again from Radhakrishna Rao (1974), we have

$$
\begin{gathered}
\sqrt{\mathrm{n}}\left(\hat{\mathrm{L}}_{\mathrm{s}}-\mathrm{L}_{\mathrm{s}}\right) \stackrel{\mathrm{d}}{\longrightarrow} \mathrm{N}\left(0, \sigma^{2}(\theta)\right) \text { as } \mathrm{n} \rightarrow \infty, \text { where } \theta=\left(\theta_{1}, \theta_{2}, \theta_{3}\right) \text { and } \\
\sigma^{2}(\theta)=\sum_{\mathrm{i}=1}^{3}\left(\frac{\partial \mathrm{L}_{\mathrm{s}}}{\partial \theta_{\mathrm{i}}}\right)^{2} \sigma_{\mathrm{ii}}=\sum_{\mathrm{i}=1}^{3}\left(\frac{\partial \mathrm{L}_{\mathrm{s}}}{\partial \theta_{\mathrm{i}}}\right)^{2} \theta_{\mathrm{i}}^{2}
\end{gathered}
$$

By substituting the partial derivatives $\left(\frac{\partial \mathrm{L}_{\mathrm{s}}}{\partial \theta_{\mathrm{i}}}\right), \mathrm{i}=1,2,3$ in (3.24), we obtain an expression for $\sigma^{2}(\theta)$. Thus, $\hat{L}_{S}$ is a CAN estimator of $L_{S}$. There are several methods for generating CAN estimators and the Method of moments and the Method of Maximum likelihood are commonly used to generate such estimators. (see Sinha(1986)).

\subsection{Confidence Limits for the Expected Number of Entities in the System}

Let $\sigma^{2}(\hat{\theta})$ be the estimator of $\sigma^{2}(\theta)$ obtained by replacing $\theta$ by a consistent estimator $\hat{\theta}$ namely $\hat{\theta}=\left(\overline{\mathrm{Y}}_{1}, \overline{\mathrm{Y}}_{2}, \overline{\mathrm{Y}}_{3}\right)$. Let $\hat{\sigma}^{2}=\sigma^{2}(\hat{\theta})$. Since $\sigma^{2}(\theta)$ is a continuous function of $\theta, \hat{\sigma}^{2}$ is a consistent estimator of $\sigma^{2}(\theta)$. i.e., $\hat{\sigma^{2}} \stackrel{P}{\longrightarrow} \sigma^{2}(\theta) \quad$ as $n \rightarrow \infty$. 
By Slutsky theorem $\left(X_{n} \stackrel{d}{\longrightarrow} X, Y_{n} \stackrel{P}{\longrightarrow} b \Rightarrow \frac{X_{n}}{Y_{n}} \stackrel{d}{\longrightarrow} \frac{X}{b}, b \neq 0\right)$, we have

$$
\sqrt{\mathrm{n}}\left(\frac{\hat{\mathrm{L}_{\mathrm{s}}}-\mathrm{L}_{\mathrm{s}}}{\hat{\sigma}}\right) \stackrel{\mathrm{d}}{\longrightarrow} \mathrm{N}(0,1) . \quad \text { i.e., } \operatorname{Pr}\left[-\mathrm{k}_{\alpha / 2}<\frac{\sqrt{\mathrm{n}\left(\hat{\mathrm{L}}_{\mathrm{s}}-\mathrm{L}_{\mathrm{s}}\right)}}{\hat{\sigma}}<\mathrm{k}_{\alpha / 2}\right]=(1-\alpha)
$$

where $\mathrm{k}_{\alpha / 2}$ is obtained from normal tables. Hence, a $100(1-\alpha) \%$ asymptotic confidence interval for $\mathrm{L}_{\mathrm{S}}$ is given by

$$
\hat{\mathrm{L}_{\mathrm{s}}} \pm \mathrm{k}_{\alpha / 2} \frac{\hat{\sigma}}{\sqrt{\mathrm{n}}}
$$

where $\hat{\sigma}$ is a consistent estimator of $\sigma(\theta)$.

\subsection{Numerical Illustration}

To study the performance of $\hat{L}_{S}$, random samples of 5000 observations are generated independently 50 times from exponential distributions assuming $\theta_{1}=2, \theta_{2}=3$ and $\theta_{3}=4$.Using the generated samples, the Maximum Likelihood estimates of $\theta_{1}, \theta_{2}$ and $\theta_{3}$ are obtained. The estimated value of $L_{S}$ i.e., $\hat{L}_{S}$ is then obtained using these estimates. Table 2 shows the calculated values of $L_{S}$ and $\hat{L}_{S}$ and the corresponding bias which is defined as the difference between the value of $\hat{L}_{S}$ and $L_{S}$.

The performance of $\hat{L}_{S}$ is measured in terms of Mean Square Error that is defined as Mean Square Error $=\frac{1}{50} \sum\left(\hat{L}_{s}-L_{S}\right)^{2}$.

The Mean Square Error value is obtained as 0.002014933. It is to be noted that values of Mean Square Error of any estimator close to zero indicate that the estimator is good. Thus, based on the numerical results, it is reasonable to conclude that the proposed estimator performs well. 
Table 2 : Values of $L_{S}$ and $\hat{L}_{S}$

\begin{tabular}{|c|c|c|c|}
\hline $\begin{array}{c}\text { Sample } \\
\cdot \\
\text { no } \\
\end{array}$ & $L_{S}$ & $\hat{L}_{S}$ & Bias \\
\hline 1 & 2.418929 & 2.342763 & -0.07617 \\
\hline 2 & 2.418929 & 2.404658 & -0.01427 \\
\hline 3 & 2.418929 & 2.416234 & -0.00269 \\
\hline 4 & 2.418929 & 2.348829 & -0.0701 \\
\hline 5 & 2.418929 & 2.402131 & -0.0168 \\
\hline 6 & 2.418929 & 2.432427 & 0.013499 \\
\hline 7 & 2.418929 & 2.37898 & -0.03995 \\
\hline 8 & 2.418929 & 2.383625 & -0.0353 \\
\hline 9 & 2.418929 & 2.392747 & -0.02618 \\
\hline 10 & 2.418929 & 2.400538 & -0.01839 \\
\hline 11 & 2.418929 & 2.354361 & -0.06457 \\
\hline 12 & 2.418929 & 2.305803 & -0.11313 \\
\hline 13 & 2.418929 & 2.308225 & -0.1107 \\
\hline 14 & 2.418929 & 2.35657 & -0.06236 \\
\hline 15 & 2.418929 & 2.368297 & -0.05063 \\
\hline 16 & 2.418929 & 2.45459 & 0.035661 \\
\hline 17 & 2.418929 & 2.430434 & 0.011505 \\
\hline 18 & 2.418929 & 2.43914 & 0.020211 \\
\hline 19 & 2.418929 & 2.39928 & -0.01965 \\
\hline 20 & 2.418929 & 2.438638 & 0.019709 \\
\hline 21 & 2.418929 & 2.351422 & -0.06751 \\
\hline 22 & 2.418929 & 2.380057 & -0.03887 \\
\hline 23 & 2.418929 & 2.359503 & -0.05943 \\
\hline 24 & 2.418929 & 2.452838 & 0.033909 \\
\hline 25 & 2.418929 & 2.44069 & 0.021761 \\
\hline
\end{tabular}

\begin{tabular}{|c|c|c|c|}
\hline $\begin{array}{c}\text { Sample } \\
\cdot \\
\text { no }\end{array}$ & $L_{S}$ & $\hat{L}_{S}$ & Bias \\
\hline 26 & 2.418929 & 2.363453 & -0.05548 \\
\hline 27 & 2.418929 & 2.448545 & 0.029616 \\
\hline 28 & 2.418929 & 2.364139 & -0.05479 \\
\hline 29 & 2.418929 & 2.365554 & -0.05338 \\
\hline 30 & 2.418929 & 2.361561 & -0.05737 \\
\hline 31 & 2.418929 & 2.40979 & -0.00914 \\
\hline 32 & 2.418929 & 2.400889 & -0.01804 \\
\hline 33 & 2.418929 & 2.41108 & -0.00785 \\
\hline 34 & 2.418929 & 2.393344 & -0.02558 \\
\hline 35 & 2.418929 & 2.386933 & -0.032 \\
\hline 36 & 2.418929 & 2.426333 & 0.007404 \\
\hline 37 & 2.418929 & 2.409509 & -0.00942 \\
\hline 38 & 2.418929 & 2.385804 & -0.03312 \\
\hline 39 & 2.418929 & 2.323868 & -0.09506 \\
\hline 40 & 2.418929 & 2.367714 & -0.05121 \\
\hline 41 & 2.418929 & 2.413293 & -0.00564 \\
\hline 42 & 2.418929 & 2.432742 & 0.013813 \\
\hline 43 & 2.418929 & 2.396278 & -0.02265 \\
\hline 44 & 2.418929 & 2.40431 & -0.01462 \\
\hline 45 & 2.418929 & 2.371189 & -0.04774 \\
\hline 46 & 2.418929 & 2.405219 & -0.01371 \\
\hline 47 & 2.418929 & 2.356025 & -0.0629 \\
\hline 48 & 2.418929 & 2.398101 & -0.02083 \\
\hline 49 & 2.418929 & 2.422104 & 0.003175 \\
\hline 50 & 2.418929 & 2.412362 & -0.00657 \\
\hline
\end{tabular}




\section{Acknowledgement}

The research of P.Chandrasekhar has been sponsored by the University Grants Commission, New Delhi. The authors thank the referees for their useful comments and suggestions, which have greatly helped in improving the paper.

\section{References}

1. Chandrasekhar, P., Chandrasekar, B. and Yadavalli, V.S.S. (2006) Statistical Inference for a tandem queue with dependent structure for service times, Proceedings of the Sixth IASTED International Conference on Modelling. Simulation and Optimization, September 11-13, 2006, Gaborone, Botswana, pp 233-238.

2. Chandrasekhar, P., Natarajan, R. and Yadavalli, V.S.S.(2008)-Statistical analysis for a tandem queue with blocking, Proceedings of the Second National Conference on Management Science and Practice (N. Ravichandran, Ed), Allied Publishers Pvt. Ltd, March 9-11,2007,Chennai, India, pp 65-72.

3. Chandrasekhar, P. and Vaidyanathan, V.S. (2009) - Statistical analysis for a two station and a three station tandem queues with blocking, Journal of Applied Statistical Science (USA), Vol. 16, No.4, pp 425 - 435.

4. Clarke, A.B. (1957) - Maximum likelihood estimates in a simple queue, Ann. Math stat., 28, pp $1036-1040$.

5. Narayan U. Bhat (2003)-Parametric Estimation in $\mathrm{M}|\mathrm{G}| 1$ and $\mathrm{GI}|\mathrm{M}| 1$ Queues using Queue Length Data, Stochastic Point Processes (S.K.Srinivasan and A. Vijaya Kumar, Eds.), Narosa Publishing House, New Delhi, pp 96-107.

6. Paul Savariappan, Chandrasekhar, P. and Vaidyanathan (2009 a) - Statistical Inference for a bulk - arrival queue, Proceedings of the $5^{\text {th }}$ Asian Mathematical Conference, Malaysia, pp 109 - 116.

7. Paul Savariappan, Chandrasekhar, P., and Mani, V. (2009 b) - Statistical Analysis for tandem and Bulk service Queueing systems, Pak. J. Statist, Vol. 25(2), pp $195-203$.

8. Radhakrishna Rao, C. (1974) - Linear Statistical Inference and its applications, Wiley Eastern Pvt. Ltd., New Delhi.

9. Tsung - Yin Wang, Jan - Chuan Ke, Kuo - Hstung Wang and Siu - Chuen Ho (2006) - Maximum likelihood estimates and confidence intervals of an $\mathrm{M} / \mathrm{M} / \mathrm{R}$ queue with heterogeneous servers, Math. Meth. Oper. Res., 63, pp $371-384$ 
10. Sinha, S.K. (1986) - Reliability and Life Testing, Wiley Eastern Pvt. Ltd., New Delhi.

11. Yadavalli, V.S.S., Adendorff, K., Erasmus, G., Chandrasekhar, P. and Deepa, S.P. (2004) - Confidence limits for expected waiting time of two queueing models, OriON (South Africa), Vol. 20, No 1, pp 1-6.

12. Yadavalli, V.S.S., Natarajan, R., and Chandrasekhar, P. (2006) Confidence limits for expected waiting time of $M|M| c \mid \infty$ and $M|M| c \mid N$ queueing models, Pak.J.Statist, Vol. 22(2), pp 171-178. 
